\title{
A Synthesis Approach to Predictive Control for Networked Control Systems
}

\author{
Yuanyuan Zou ${ }^{1}$, James Lam $^{2}$, Yugang Niu ${ }^{1}$, Dewei $\mathbf{L i}^{3}$, \\ 1. Key Laboratory of Advanced Control and Optimization for Chemical Process, Ministry of Education, East China University of Science \\ and Technology, Shanghai, 200237, China \\ E-mail: yyzou@ecust.edu.cn, acniuyg@ecust.edu.cn \\ 2. Department of Mechanical Engineering, The University of Hong Kong, Hong Kong \\ E-mail: james.lam@hku.hk \\ 3. Department of Automation, Shanghai Jiao Tong University, Shanghai, 200240, China \\ E-mail: dwli@sjtu.edu.cn
}

\begin{abstract}
This paper studies a synthesis approach to predictive control for networked control systems with data loss and quantization. An augmented Markov jump linear model with polytopic uncertainties is modeled to describe the quantization errors and possible data loss. Based on this model, a predictive control synthesis approach is developed, which involves online optimization of a infinite horizon objective and conditions to deal with system constraints. The proposed MPC algorithm guarantees closed-loop mean-square stability and constraints satisfaction.
\end{abstract}

Key Words: Predictive Control, Networked Control Systems, Stability

\section{Introduction}

With the development of large-scale or complex industrial systems, communication networks play a more and more important role. They bring advantages for control systems, such as low cost, high flexibility, simple installation and maintenance. However, owing to the limited communication capacities, the insertion of a communication network also has some detrimental effects on practical feedback control systems [1], [10], [13]. The signals are usually quantized before being transmitted. Moreover, in the process of transmission, the quantized signals may be lost. Thus, the performance of controlled systems will inevitably be subjected to the effects of quantization error and data loss, so that the conventional control methods may not work effectively. Hence, this paper focuses on the problem of control over above network environment.

A lot of effort has recently been made on the design and analysis of control systems with quantization or/and data loss, see for example, [4], [5], [8], [11], [14], [16]. In [8] and [11], logarithmic quantizer was introduced and proven that quantized stabilization is equivalent to the robust stabilization of an associated system with sector bound uncertainty. Following [8] and [11], different control approaches to deal with quantized stabilization problem have been studied in [4], [5], [14], [16]. For data loss, it can be often and appropriately modeled as random processes described by a probability distribution. A simple stochastic approach is to describe it as an independent and identically distributed (i.i.d.) Bernoulli process [3], [12], [15], [17]. To describe data loss process more accurately, Markov jump process was adopted [6], [7], [9]. In [6], a discrete-time Markov chain with known transition probability matrix was used to model the data loss process and the stabilization problem was inves-

This work is partially supported by GRF HKU 7140/11E, National Natural Science Foundation of China $(61273073$, 61374107, 61374110), the Fundamental Research Funds for the Central Universities, and the Cheung Kong Chair Professor Program. tigated. It was considered in [7] the stability of sampled-data networked linear systems with Markovian packet losses, and the state estimation problem was studied in [9].

Model predictive control (MPC), also known as receding horizon control, has received much attention in the past decades due to its extensive applications. It can incorporate the input/output constraints into the on-line optimization and achieve approximately optimal control performance, which inspires the development of MPC for networked control systems. In [2] and [19], robust MPC strategies were presented to stabilize the quantized feedback control systems. In [18], Bernoulli data loss was assumed and a predictive control law involving missing probability was designed to stabilize the closed-loop system in mean square sense. However, to the authors' best knowledge, the predictive control synthesis problem for networked control systems with both quantization and Markovian data loss remains open.

Motivated by the above discussion, this work considers the synthesis problem for control systems where controller output data is transmitted over a communication network. Both Markovian data loss and logarithmic quantizers are considered in the network. A novel compensation strategy is introduced to deal with the multiple data loss and the influence of quantization errors can be viewed as polytopic uncertainties of control systems. Then a robust probabilitybased predictive control synthesis approach is proposed and the system constraints are satisfied in spite of multiple data loss and quantization errors.

The organization of this paper is as follows. Section 2 introduces the modeling of networked control systems with quantization and data loss. In Section 3, a predictive synthesis approach is presented, and the closed-loop stability results are achieved. Some conclusions are drawn in Section 4. 
The notations used throughout this paper are fairly standard: $\mathbf{R}^{n}$ denotes the $n$-dimensional Euclidean space. $P>0(\geq 0)$ means that $P$ is real symmetric and positive definite (semi-definite). In block symmetric matrices, we use an asterisk $(*)$ to represent a term that is induced by symmetry. $\operatorname{Diag}\{\cdots\}$ stands for a block-diagonal matrix and $\operatorname{Co}\{\cdots\}$ denotes the convex hull, that is, if $\Omega=\operatorname{Co}\left\{A_{1}, A_{2}, \ldots, A_{L}\right\}=$ $\left\{\sum_{i=1}^{L} a_{i} A_{i} \mid \sum_{i=1}^{L} a_{i}=1, a_{i} \geq 0\right\} . \mathcal{E}$ is the expectation operator and $\mathcal{E}_{x}$ denotes conditional expectation with respect to $x$. The notation $\psi(k+j \mid k)$ (where $\psi$ may represent $u, x$ or $z$ ) denotes the prediction of $\psi$ at future time $k+j$ based on the current state $x(k)$.

\section{Problem Formulation}

Consider the following system:

$$
x(k+1)=A x(k)+B u(k),
$$

where $u(k) \in \mathbf{R}^{m}$ is the control input, $x(k) \in \mathbf{R}^{n}$ is the state vector, and the constraints on the control input are:

$$
\left|u_{j}(k)\right| \leq \bar{u}_{j}, \quad j=1, \ldots, m .
$$

We assume that the communication network is situated between the controller output and the plant input. Thus, all the data to be transmitted need to be quantized and may be lost. The quantizer at time instant $k$ is modeled by

$$
u(k)=f(v(k)),
$$

where $f(\cdot)$ is a logarithmic quantizer with the following form:

$$
f(v)=\left\{\begin{array}{cl}
\nu_{i}, & \text { if } \frac{1}{1+\tau} \nu_{i}<v \leq \frac{1}{1-\tau} \nu_{i}, \quad v>0 \\
0, & \text { if } v=0 \\
-f(-v), & \text { if } v<0
\end{array}\right.
$$

The set of quantized levels is characterized by

$$
\begin{gathered}
\mathcal{V}=\left\{ \pm \nu_{i}, \nu_{i}=\rho^{i} \nu_{0}, i= \pm 1, \pm 2, \ldots\right\} \cup\left\{ \pm \nu_{0}\right\} \cup\{0\} \\
0<\rho<1, \quad \nu_{0}>0
\end{gathered}
$$

where

$$
\rho=\frac{1-\tau}{1+\tau} .
$$

From [8], for the quantized control signals with individual quantizer $f_{l}$ for channel $l(l=1, \ldots, m)$, a sector-bound expression can be given as follows:

$$
\begin{aligned}
u(k) & =\left[\begin{array}{llll}
f_{1}\left(v_{1}(k)\right) & f_{2}\left(v_{2}(k)\right) & \cdots & f_{m}\left(v_{m}(k)\right)
\end{array}\right]^{T} \\
& =\Lambda(k) v(k),
\end{aligned}
$$

where

$$
\begin{aligned}
\Lambda(k)= & \operatorname{diag}\left\{1+\xi_{1}(k), 1+\xi_{2}(k), \ldots, 1+\xi_{m}(k)\right\} \\
& \xi_{l}(k) \in\left[-\tau_{l}, \tau_{l}\right], \quad l=1, \ldots, m .
\end{aligned}
$$

From (7), $\Lambda(k)$ can be represented by

$$
\Lambda(k) \in \Omega=\operatorname{Co}\left\{\Lambda^{(1)}, \Lambda^{(2)}, \ldots, \Lambda^{\left(2^{m}\right)}\right\},
$$

that is,

$$
\Lambda(k)=\sum_{i=1}^{2^{m}} a_{i}(k) \Lambda^{(i)}, \quad \sum_{i=1}^{2^{m}} a_{i}(k)=1, \quad a_{i}(k) \geq 0,
$$

where $\Lambda^{(i)}$ is a diagonal matrix with entries being $1-\tau_{l}$ or $1+\tau_{l}$, and the $2^{m}$ combinations of $1-\tau_{l}$ and $1+\tau_{l}$ form all $\Lambda^{(i)}$.

When the data is transmitted from the controller to the actuator, it may be lost. Here a stochastic variable $\gamma(k) \in \mathbf{R}$ is introduced to denote the data status for time instant $k$ (1 for transmitted data, 0 for missing data). The data status process is assumed to be a discrete-time homogeneous Markov chain taking values in a finite set $\mathcal{W}=\{0,1\}$ with transition probability matrix

$$
\Pi=\left[\begin{array}{cc}
1-\beta & \beta \\
\alpha & 1-\alpha
\end{array}\right]
$$

where

$$
\begin{gathered}
0 \leq \operatorname{Pr}(\gamma(k+1)=0 \mid \gamma(k)=1)=\alpha \leq 1 \\
0 \leq \operatorname{Pr}(\gamma(k+1)=1 \mid \gamma(k)=0)=\beta \leq 1
\end{gathered}
$$

Hence, $\alpha$ and $\beta$ are called the failure probability and the recovery probability, respectively.

The following compensation strategy is introduced to deal with the negative effects caused by multiple data loss:

$$
u_{d}(k)= \begin{cases}u(k), & \text { if } \gamma(k)=1 ; \\ \delta u_{d}(k-1), & \text { if } \gamma(k)=0,\end{cases}
$$

that is,

$$
u_{d}(k)=\gamma(k) u(k)+(1-\gamma(k)) \delta u_{d}(k-1) .
$$

In (13), $\delta \in(0,1]$ is a forgetting factor, which can improve the flexibility of compensation strategy. It is worth noting that $\delta=1$ means hold-input, that is, the latest control input stored in the actuator buffer is used when the data packet is lost.

Taking into account of the practical realization of compensation strategy (13), the following assumptions are made:

- The data may be lost successively (that is, multiple data loss), and assume that the maximum data loss upper bound is $\theta_{\max }$.

- At each time instant $k$, the control input $u_{d}(k-1)$ and the current state $x(k)$ are transmitted to controller together.

Based on (1), (3) and (13), the closed-loop dynamic model (1) is rewritten as

$$
x(k+1)=A x(k)+\gamma(k) B \Lambda(k) v(k)+(1-\gamma(k)) \delta B u_{d}(k-1) .
$$

Then, the objective is to synthesize a predictive controller to drive the closed-loop system from any state to the origin in the mean square sense by minimizing a performance objective function. 


\section{Predictive Control Synthesis}

In this section, we will develop a MPC for networked control systems with quantization and data loss. The following state feedback predictive control law is utilized:

$$
v(k+i \mid k)=F x(k+i \mid k) .
$$

Let

$$
z(k)=\left[\begin{array}{c}
x(k) \\
u_{d}(k-1)
\end{array}\right],
$$

then the augmented model of (14) and (15) is

$$
z(k+1)=\left[\begin{array}{cc}
A+\gamma(k) B \Lambda(k) F & (1-\gamma(k)) \delta B \\
\gamma(k) \Lambda(k) F & (1-\gamma(k)) \delta I
\end{array}\right] z(k),
$$

which is an uncertain system dependent on the stochastic variable $\gamma(k)$.

At each time $k$, the following performance objective function of the MPC synthesis problem is defined:

$$
\min _{u(k+i \mid k)} \max _{\Lambda(k+i \mid k)} J_{\infty}(k),
$$

where

$$
\begin{array}{r}
J_{\infty}(k)=\sum_{i=0}^{\infty} \mathcal{E}_{z(k)}\left\{z^{T}(k+i \mid k) S z(k+i \mid k)\right. \\
\left.+u_{d}^{T}(k+i \mid k) R u_{d}(k+i \mid k)\right\},
\end{array}
$$

$S=\operatorname{diag}\left\{S_{1}, 0\right\}$, with $S_{1}>0, R>0$.

In order to derive an upper bound on the performance objective (19), we choose the quadratic Lyapunov function candidate

$$
\begin{aligned}
& V(k+i \mid k) \\
= & z^{T}(k+i \mid k)\left[\begin{array}{cc}
M_{\gamma(k+i \mid k)} & 0 \\
0 & N_{\gamma(k+i \mid k)}
\end{array}\right] z(k+i \mid k), \\
\gamma(k+i \mid k) & \in \mathcal{W}=\{0,1\}
\end{aligned}
$$

and suppose $V(\cdot)$ satisfies the following contractiveness condition:

$$
\begin{aligned}
& \mathcal{E}_{z(k)}\{V(k+i+1 \mid k)-V(k+i \mid k)\} \\
\leq \quad & -\mathcal{E}_{z(k)}\left\{z^{T}(k+i \mid k) S z(k+i \mid k)\right. \\
& \left.+u_{d}^{T}(k+i \mid k) R u_{d}(k+i \mid k)\right\},
\end{aligned}
$$

where $M_{\gamma(k+i \mid k)} \in \mathbf{R}^{n \times n}$ and $N_{\gamma(k+i \mid k)} \in \mathbf{R}^{m \times m}$ are positive definite matrices.

Then, a sufficient condition for the satisfaction of (21) is presented.

Lemma 1. The contractiveness condition (21) is satisfied if there exist a scalar $\varepsilon>0$, symmetric matrices $\bar{M}_{0}, \bar{N}_{0}, \bar{M}_{1}$, $\bar{N}_{1}, \bar{W}_{0}, \bar{T}_{0}, \bar{W}_{1}, \bar{T}_{1}$ and any matrix $Y_{1}=F \bar{M}_{1}$, such that the following matrix inequalities (22)-(25) hold.

$$
\left[\begin{array}{cccccc}
\bar{M}_{0} & * & * & * & * & * \\
0 & \bar{N}_{0} & * & * & * & * \\
A \bar{M}_{0} & \delta B \bar{N}_{0} & \bar{W}_{0} & * & * & * \\
0 & \delta \bar{N}_{0} & 0 & \bar{T}_{0} & * & * \\
\bar{M}_{0} & 0 & 0 & 0 & \varepsilon S_{1}^{-1} & * \\
0 & \delta \bar{N}_{0} & 0 & 0 & 0 & \varepsilon R^{-1}
\end{array}\right] \geq 0,(22)
$$

$$
\left[\begin{array}{cccccc}
\bar{M}_{1} & * & * & * & * & * \\
0 & \bar{N}_{1} & * & * & * & * \\
A \bar{M}_{1}+B \Lambda^{(l)} Y_{1} & 0 & \bar{W}_{1} & * & * & * \\
\Lambda^{(l)} Y_{1} & 0 & 0 & \bar{T}_{1} & * & * \\
\bar{M}_{1} & 0 & 0 & 0 & \varepsilon S_{1}^{-1} & * \\
\Lambda^{(l)} Y_{1} & 0 & 0 & 0 & 0 & \varepsilon R^{-1}
\end{array}\right] \geq 0
$$

$$
\left[\begin{array}{cccccc}
\bar{W}_{1} & * & * & * & * & * \\
0 & \bar{T}_{1} & * & * & * & * \\
\alpha^{\frac{1}{2}} \bar{W}_{1} & 0 & \bar{M}_{0} & * & * & * \\
(1-\alpha)^{\frac{1}{2}} \bar{W}_{1} & 0 & 0 & \bar{M}_{1} & * & * \\
0 & \alpha^{\frac{1}{2}} \bar{T}_{1} & 0 & 0 & \bar{N}_{0} & * \\
0 & (1-\alpha)^{\frac{1}{2}} \bar{T}_{1} & 0 & 0 & 0 & \bar{N}_{1}
\end{array}\right] \geq 0
$$

$$
\left[\begin{array}{cccccc}
\bar{W}_{0} & * & * & * & * & * \\
0 & \bar{T}_{0} & * & * & * & * \\
(1-\beta)^{\frac{1}{2}} \bar{W}_{0} & 0 & \bar{M}_{0} & * & * & * \\
\beta^{\frac{1}{2}} \bar{W}_{0} & 0 & 0 & \bar{M}_{1} & * & * \\
0 & (1-\beta)^{\frac{1}{2}} \bar{T}_{0} & 0 & 0 & \bar{N}_{0} & * \\
0 & \beta^{\frac{1}{2}} \bar{T}_{0} & 0 & 0 & 0 & \bar{N}_{1}
\end{array}\right] \geq 0 .
$$

$$
\begin{aligned}
& z(k+i+1 \mid k)=\left[\begin{array}{cc}
A+\gamma(k+i \mid k) B \Lambda(k+i \mid k) F & (1-\gamma(k+i \mid k)) \delta B \\
\gamma(k+i \mid k) \Lambda(k+i \mid k) F & (1-\gamma(k+i \mid k)) \delta I
\end{array}\right] z(k+i \mid k), \\
& \mathcal{E}_{z(k)}\{V(k+i+1 \mid k)-V(k+i \mid k)\} \\
& =\mathcal{E}_{z(k)}\left\{\mathcal { E } _ { z ( k + i | k ) } \left\{z^{T}(k+i \mid k)\left[\begin{array}{cc}
A+\gamma(k+i \mid k) B \Lambda(k+i \mid k) F & (1-\gamma(k+i \mid k)) \delta B \\
\gamma(k+i \mid k) \Lambda(k+i \mid k) F & (1-\gamma(k+i \mid k)) \delta I
\end{array}\right]^{T}\right.\right. \\
& \times\left[\begin{array}{cc}
M_{(\gamma(k+i+1 \mid k) \mid \gamma(k+i \mid k))} & 0 \\
0 & N_{(\gamma(k+i+1 \mid k) \mid \gamma(k+i \mid k))}
\end{array}\right]\left[\begin{array}{cc}
A+\gamma(k+i \mid k) B \Lambda(k+i \mid k) F & (1-\gamma(k+i \mid k)) \delta B \\
\gamma(k+i \mid k) \Lambda(k+i \mid k) F & (1-\gamma(k+i \mid k)) \delta I
\end{array}\right] \\
& \left.\times z(k+i \mid k)\}-z^{T}(k+i \mid k)\left[\begin{array}{cc}
M_{\gamma(k+i \mid k)} & 0 \\
0 & N_{\gamma(k+i \mid k)}
\end{array}\right] z(k+i \mid k)\right\}, \\
& \mathcal{E}_{\gamma(k+i \mid k)=1}\left\{\left[\begin{array}{cc}
M_{(\gamma(k+i+1 \mid k) \mid \gamma(k+i \mid k)=1)} & 0 \\
0 & N_{(\gamma(k+i+1 \mid k) \mid \gamma(k+i \mid k)=1)}
\end{array}\right]\right\} \\
& =\left[\begin{array}{cc}
\alpha M_{0} & 0 \\
0 & \alpha N_{0}
\end{array}\right]+\left[\begin{array}{cc}
(1-\alpha) M_{1} & 0 \\
0 & (1-\alpha) N_{1}
\end{array}\right] \text {. }
\end{aligned}
$$


Proof. Based on augmented model (16)-(17) and the quadratic Lyapunov function defined in (20), we can get (26) and (27).

For $\gamma(k+i \mid k)=1$, taking the transition probabilities in (10) into account, it is obvious that (28) holds. Then, the contractiveness condition (21) can be written as

$$
\begin{aligned}
& \mathcal{E}_{z(k)}\{V(k+i+1 \mid k)-V(k+i \mid k)\}+\mathcal{E}_{z(k)}\left\{z^{T}(k+i \mid k)\right. \\
& \left.\times S z(k+i \mid k)+u_{d}^{T}(k+i \mid k) R u_{d}(k+i \mid k)\right\} \\
& =\mathcal{E}_{z(k)}\left\{z^{T}(k+i \mid k)\left[\begin{array}{cc}
A+B \Lambda(k+i \mid k) F & 0 \\
\Lambda(k+i \mid k) F & 0
\end{array}\right]^{T}\right. \\
& \times\left(\left[\begin{array}{cc}
\alpha M_{0} & 0 \\
0 & \alpha N_{0}
\end{array}\right]+\left[\begin{array}{cc}
(1-\alpha) M_{1} & 0 \\
0 & (1-\alpha) N_{1}
\end{array}\right]\right) \\
& \times\left[\begin{array}{cc}
A+B \Lambda(k+i \mid k) F & 0 \\
\Lambda(k+i \mid k) F & 0
\end{array}\right] z(k+i \mid k) \\
& \left.-z^{T}(k+i \mid k)\left[\begin{array}{cc}
M_{1} & 0 \\
0 & N_{1}
\end{array}\right] z(k+i \mid k)\right\} \\
& +\mathcal{E}_{z(k)}\left\{z^{T}(k+i \mid k)\left[\begin{array}{cc}
S_{1} & 0 \\
0 & 0
\end{array}\right] z(k+i \mid k)\right. \\
& +z^{T}(k+i \mid k)\left[\begin{array}{c}
F^{T} \Lambda^{T}(k+i \mid k) \\
0
\end{array}\right] R \\
& \left.\times\left[\begin{array}{c}
\Lambda(k+i \mid k) F \\
0
\end{array}\right] z(k+i \mid k)\right\} \leq 0,
\end{aligned}
$$

which can be satisfied if the following two inequalities hold:

$$
\begin{aligned}
& {\left[\begin{array}{cc}
A+B \Lambda(k+i \mid k) F & 0 \\
\Lambda(k+i \mid k) F & 0
\end{array}\right]^{T}\left[\begin{array}{cc}
W_{1} & 0 \\
0 & T_{1}
\end{array}\right] } \\
\times & {\left[\begin{array}{cc}
A+B \Lambda(k+i \mid k) F & 0 \\
\Lambda(k+i \mid k) F & 0
\end{array}\right]-\left[\begin{array}{cc}
M_{1} & 0 \\
0 & N_{1}
\end{array}\right] } \\
+ & {\left[\begin{array}{cc}
S_{1} & 0 \\
0 & 0
\end{array}\right]+\left[\begin{array}{c}
F^{T} \Lambda^{T}(k+i \mid k) \\
0
\end{array}\right] R } \\
\times & {\left[\begin{array}{c}
F^{T} \Lambda^{T}(k+i \mid k) \\
0
\end{array}\right]^{T} } \\
\leq & {\left[\begin{array}{cc}
\alpha M_{0} & 0 \\
0 & \alpha N_{0}
\end{array}\right]+\left[\begin{array}{cc}
(1-\alpha) M_{1} & 0 \\
0 & (1-\alpha) N_{1}
\end{array}\right] } \\
\leq & {\left[\begin{array}{cc}
W_{1} & 0 \\
0 & T_{1}
\end{array}\right] . }
\end{aligned}
$$

Pre- and post-multiplying (30) and (31) by $\operatorname{diag}\left\{\varepsilon^{\frac{1}{2}} M_{1}^{-1}, \varepsilon^{\frac{1}{2}} N_{1}^{-1}\right\}$ and $\operatorname{diag}\left\{W_{1}^{-1}, T_{1}^{-1}\right\}$, respectively, and applying Schur's complement with

$$
\begin{aligned}
& \varepsilon M_{1}^{-1}=\bar{M}_{1}, \quad \varepsilon N_{1}^{-1}=\bar{N}_{1}, \quad \varepsilon M_{0}^{-1}=\bar{M}_{0}, \\
& \varepsilon N_{0}^{-1}=\bar{N}_{0}, \quad \varepsilon W_{1}^{-1}=\bar{W}_{1}, \quad \varepsilon T_{1}^{-1}=\bar{T}_{1}, \\
& Y_{1}=F \bar{M}_{1},
\end{aligned}
$$

we can obtain (24) and (33). Since

$$
\Lambda(k+i \mid k) \in \operatorname{Co}\left\{\Lambda^{(1)}, \Lambda^{(2)}, \ldots, \Lambda^{\left(2^{m}\right)}\right\},
$$

inequality (33) can be transformed into (23).

$$
\text { For } \gamma(k+i \mid k)=0 \text {, we have }
$$

$$
\begin{aligned}
\mathcal{E}_{z(k)}\{ & V(k+i+1 \mid k)-V(k+i \mid k)\}+\mathcal{E}_{z(k)}\left\{z^{T}(k+i \mid k)\right. \\
& \left.\times S z(k+i \mid k)+u_{d}^{T}(k+i \mid k) R u_{d}(k+i \mid k)\right\} \\
= & \mathcal{E}_{z(k)}\left\{z^{T}(k+i \mid k)\left[\begin{array}{cc}
A & \delta B \\
0 & \delta I
\end{array}\right]^{T}\left[\begin{array}{cc}
W_{0} & 0 \\
0 & T_{0}
\end{array}\right]\right. \\
& {\left[\begin{array}{cc}
A & \delta B \\
0 & \delta I
\end{array}\right] z(k+i \mid k)-z^{T}(k+i \mid k) } \\
& \left.\times\left[\begin{array}{cc}
M_{0} & 0 \\
0 & N_{0}
\end{array}\right] z(k+i \mid k)\right\}+\mathcal{E}_{z(k)}\left\{z^{T}(k+i \mid k)\right. \\
& \times\left[\begin{array}{cc}
S_{1} & 0 \\
0 & 0
\end{array}\right] z(k+i \mid k)+z^{T}(k+i \mid k)\left[\begin{array}{c}
0 \\
\delta I
\end{array}\right] \\
& \left.\times R\left[\begin{array}{cc}
0 & \delta I
\end{array}\right] z(k+i \mid k)\right\} \leq 0, \\
& {\left[\begin{array}{cc}
1-\beta) M_{0} \\
0
\end{array}\right]+\left[\begin{array}{cc}
\beta M_{1} & 0 \\
0 & \beta N_{1}
\end{array}\right] } \\
& \leq\left[\begin{array}{cc}
W_{0} & 0 \\
0 & T_{0}
\end{array}\right] .
\end{aligned}
$$

By similar procedure with $\varepsilon W_{0}^{-1}=\bar{W}_{0}, \varepsilon T_{0}^{-1}=\bar{T}_{0}$, we can get (22) and (25). The proof is completed.

It is obvious that if the contractiveness condition (21) holds, $\lim _{i \rightarrow \infty} \mathcal{E}_{z(k)} z(k+i \mid k)=0$. Thus, summing (21) from $i=0$ to $i=\infty$, we can get an upper bound on the control performance $J_{\infty}(k)$ :

$$
\max _{\Lambda(k+i \mid k)} J_{\infty}(k) \leq V(k \mid k) .
$$

If Lemma 1 is satisfied, then $V(k \mid k) \leq \varepsilon$ if and only if the following LMIs hold:

$$
\begin{aligned}
& {\left[\begin{array}{ccc}
1 & * & * \\
x(k) & \bar{M}_{1} & * \\
u_{d}(k-1) & 0 & \bar{N}_{1}
\end{array}\right] \geq 0,} \\
& {\left[\begin{array}{ccc}
1 & * & * \\
x(k) & \bar{M}_{0} & * \\
u_{d}(k-1) & 0 & \bar{N}_{0}
\end{array}\right] \geq 0 .}
\end{aligned}
$$

Now we will show how to satisfy the input constraints (2) in the presence of data loss and quantization. In view of the possible data status, the following two cases are considered.

(I). Data can be transmitted from the controller to the actuator successively. In order to satisfy the input constraints, an additional condition should be imposed as follows:

$$
V(k+i+1 \mid k)<V(k+i \mid k),
$$

$$
\{\gamma(k+i \mid k)=1, \gamma(k+i+1 \mid k)=1\} .
$$

$$
\left[\begin{array}{cccccc}
\bar{M}_{1} & * & * & * & * & * \\
0 & \bar{N}_{1} & * & * & * & * \\
A \bar{M}_{1}+B \Lambda(k+i \mid k) Y_{1} & 0 & \bar{W}_{1} & * & * & * \\
\Lambda(k+i \mid k) Y_{1} & 0 & 0 & \bar{T}_{1} & * & * \\
\bar{M}_{1} & 0 & 0 & 0 & \varepsilon S_{1}^{-1} & * \\
\Lambda(k+i \mid k) Y_{1} & 0 & 0 & 0 & 0 & \varepsilon R^{-1}
\end{array}\right] \geq 0
$$


(II). Data loss occurs in the transmission from the controller to the actuator. The following conditions should be incorporated into the input constraints:

$$
\begin{gathered}
V(k+i+1 \mid k)<V(k+i \mid k), \\
\{\gamma(k+i \mid k)=1, \gamma(k+i+1 \mid k)=0\}, \\
V(k+i+h+1 \mid k)<\varepsilon, \\
\{\gamma(k+i \mid k)=1, \gamma(k+i+1 \mid k)=0, \ldots, \\
\gamma(k+i+h \mid k)=0, \gamma(k+i+h+1 \mid k)=1, \\
h=\{1, \ldots, \bar{\theta}(k)\}\} \\
V(k+i+h \mid k)<\varepsilon, \\
\{\gamma(k+i \mid k)=0, \ldots, \gamma(k+i+h-1 \mid k)=0, \\
\gamma(k+i+h \mid k)=1, h=\{1, \ldots, \bar{\theta}(k)\}\},
\end{gathered}
$$

where $h$ represents the number of successive data loss.

Lemma 2. The constraints (40)-(43) are satisfied if there exist a scalar $\varepsilon>0$, symmetric matrices $\bar{M}_{1}, \bar{N}_{1}, \bar{M}_{0}, \bar{N}_{0}, \bar{M}_{1, s}, \bar{N}_{1, s}, \bar{M}_{0, s}, \bar{N}_{0, s}, \quad(s \quad=$ $1, \ldots, \bar{\theta}(k)-1)$ and matrix $Y_{1}$ satisfying the following LMIs:

$$
\begin{aligned}
& {\left[\begin{array}{cccc}
\bar{M}_{1} & * & * & * \\
0 & \bar{N}_{1} & * & * \\
A \bar{M}_{1}+B \Lambda^{(l)} Y_{1} & 0 & \bar{M}_{1} & * \\
\Lambda^{(l)} Y_{1} & 0 & 0 & \bar{N}_{1}
\end{array}\right] \geq 0} \\
& l=1, \ldots, 2^{m}, \\
& {\left[\begin{array}{cccc}
\bar{M}_{1} & * & * & * \\
0 & \bar{N}_{1} & * & * \\
A \bar{M}_{1}+B \Lambda^{(l)} Y_{1} & 0 & \bar{M}_{0} & * \\
\Lambda^{(l)} Y_{1} & 0 & 0 & \bar{N}_{0}
\end{array}\right] \geq 0} \\
& l=1, \ldots, 2^{m}, \\
& {\left[\begin{array}{cccc}
\bar{M}_{0} & * & * & * \\
0 & \bar{N}_{0} & * & * \\
A \bar{M}_{0} & \delta B \bar{N}_{0} & \bar{M}_{1} & * \\
0 & \delta \bar{N}_{0} & 0 & \bar{N}_{1}
\end{array}\right] \geq 0} \\
& {\left[\begin{array}{cccc}
\bar{M}_{1, s} & * & * & * \\
0 & \bar{N}_{1, s} & * & * \\
A \bar{M}_{1, s} & \delta B \bar{N}_{1, s} & \bar{M}_{1, s+1} & * \\
0 & \delta \bar{N}_{1, s} & 0 & \bar{N}_{1, s+1}
\end{array}\right] \geq 0} \\
& s=1, \ldots, \bar{\theta}(k)-2 \text {, } \\
& {\left[\begin{array}{cccc}
\bar{M}_{1, s} & * & * & * \\
0 & \bar{N}_{1, s} & * & * \\
A \bar{M}_{1, s} & \delta B \bar{N}_{1, s} & \bar{M}_{1} & * \\
0 & \delta \bar{N}_{1, s} & 0 & \bar{N}_{1}
\end{array}\right] \geq 0} \\
& s=1, \ldots, \bar{\theta}(k)-1 \text {, } \\
& {\left[\begin{array}{cccc}
\bar{M}_{0, s} & * & * & * \\
0 & \bar{N}_{0, s} & * & * \\
A \bar{M}_{0, s} & \delta B \bar{N}_{0, s} & \bar{M}_{0, s+1} & * \\
0 & \delta \bar{N}_{0, s} & 0 & \bar{N}_{0, s+1}
\end{array}\right] \geq 0} \\
& s=1, \ldots, \bar{\theta}(k)-2 \text {, } \\
& {\left[\begin{array}{cccc}
\bar{M}_{0, s} & * & * & * \\
0 & \bar{N}_{0, s} & * & * \\
A \bar{M}_{0, s} & \delta B \bar{N}_{0, s} & \bar{M}_{1} & * \\
0 & \delta \bar{N}_{0, s} & 0 & \bar{N}_{1}
\end{array}\right] \geq 0} \\
& s=1, \ldots, \bar{\theta}(k)-1 \text {, }
\end{aligned}
$$

$$
\begin{aligned}
& {\left[\begin{array}{cccc}
\bar{M}_{0} & * & * & * \\
0 & \bar{N}_{0} & * & * \\
A \bar{M}_{0} & \delta B \bar{N}_{0} & \bar{M}_{1,1} & * \\
0 & \delta \bar{N}_{0} & 0 & \bar{N}_{1,1}
\end{array}\right] \geq 0, \quad \bar{\theta}(k) \geq 2,} \\
& {\left[\begin{array}{cccc}
\bar{M}_{0} & * & * & * \\
0 & \bar{N}_{0} & * & * \\
A \bar{M}_{0} & \delta B \bar{N}_{0} & \bar{M}_{0,1} & * \\
0 & \delta \bar{N}_{0} & 0 & \bar{N}_{0,1}
\end{array}\right] \geq 0, \bar{\theta}(k) \geq 2 .}
\end{aligned}
$$

Proof. If the data can be transmitted successively, based on augmented model (17) and similar procedure in Lemma 1, inequality (40) is satisfied if and only if (44) holds.

For the number of successive data loss $h=1$, the possible loss modes are

$$
\begin{aligned}
& \{\gamma(k+i \mid k)=1, \gamma(k+i+1 \mid k)=0, \gamma(k+i+2 \mid k)=1\} \\
& \text { or }\{\gamma(k+i \mid k)=0, \gamma(k+i+1 \mid k)=1\} .
\end{aligned}
$$

According to the compensation strategy (13), inequalities (41)-(43) can be transformed into (45)-(46).

For the number of successive data loss $h=2$, the possible loss modes are

$\{\gamma(k+i \mid k)=1, \gamma(k+i+1 \mid k)=0, \gamma(k+i+2 \mid k)=0$,

$\gamma(k+i+3 \mid k)=1\}$, i.e., $1 \rightarrow 0 \rightarrow 0 \rightarrow 1$;

$\{\gamma(k+i \mid k)=0, \gamma(k+i+1 \mid k)=0, \gamma(k+i+2 \mid k)=1\}$,

i.e., $1 \rightarrow 0 \rightarrow 0 \rightarrow 1$.

For the case $1 \rightarrow 0 \rightarrow 0 \rightarrow 1$, the corresponding Lyapunov functions are

$$
\begin{aligned}
& V(k+i+1 \mid k) \\
= & z^{T}(k+i+1 \mid k)\left[\begin{array}{cc}
M_{0} & 0 \\
0 & N_{0}
\end{array}\right] z(k+i+1 \mid k), \\
& V(k+i+2 \mid k) \\
= & z^{T}(k+i+2 \mid k)\left[\begin{array}{cc}
M_{1}^{1} & 0 \\
0 & N_{1}^{1}
\end{array}\right] z(k+i+2 \mid k), \\
& V(k+i+3 \mid k) \\
= & z^{T}(k+i+3 \mid k)\left[\begin{array}{cc}
M_{1} & 0 \\
0 & N_{1}
\end{array}\right] z(k+i+3 \mid k) .
\end{aligned}
$$

According to augmented model (17) and Schur's complement, the constraints in (41) and (42) can be satisfied by transforming inequalities (45), (48) and (51) with $\bar{M}_{1}=$ $\varepsilon M_{1}^{-1}, \bar{N}_{1}=\varepsilon N_{1}^{-1}, \bar{M}_{0}=\varepsilon M_{0}^{-1}, \bar{N}_{0}=\varepsilon N_{0}^{-1}, \bar{M}_{1}^{1}=$ $\varepsilon\left(M_{1}^{1}\right)^{-1}, \bar{N}_{1}^{1}=\varepsilon\left(N_{1}^{1}\right)^{-1}$. Similarly, from (50) and (52), we can get (43).

For $h=3, \ldots, \bar{\theta}(k)$, by similar procedure and together with (47) and (49), inequalities (41)-(43) are satisfied. Here we omit it for brevity.

Base on the above discussions, we can incorporate the system constraints into our constrained MPC optimization problems as follows.

Lemma 3. The input constraints in (2) can be satisfied if LMIs (38)-(39), (44)-(52) are feasible and

$$
\begin{aligned}
{\left[\begin{array}{cc}
Z & \Lambda^{(l)} Y_{1} \\
* & \bar{M}_{1}
\end{array}\right] } & \geq 0, \quad Z_{j j} \leq \bar{u}_{j}^{2} \\
l & =1, \ldots, 2^{m}, \quad j=1, \ldots, m,(53)
\end{aligned}
$$

where $Z_{j j}$ is the $i$-th diagonal element of $Z$. 
Proof. For constraint (2), it is imposed on the present and the entire horizon of future manipulated variables, although only the first control move $u(k \mid k)$ is implemented, that is,

$$
\left|u_{j}(k+i \mid k)\right| \leq \bar{u}_{j}, \quad i \geq 0 \text {. }
$$

If data can be transmitted successively, applying (38) and (40), it follows that

$$
\begin{aligned}
& {\left[\begin{array}{c}
x(k+i \mid k) \\
u_{d}(k+i-1 \mid k)
\end{array}\right]^{T}\left[\begin{array}{cc}
\bar{M}_{1}^{-1} & 0 \\
0 & \bar{N}_{1}^{-1}
\end{array}\right]} \\
& \times\left[\begin{array}{c}
x(k+i \mid k) \\
u_{d}(k+i-1 \mid k)
\end{array}\right] \leq 1 .
\end{aligned}
$$

Then, we have

$$
\begin{aligned}
\left|u_{j}(k+i \mid k)\right|^{2} & =\left|\psi_{j} \Lambda(k+i) Y_{1} \bar{M}_{1}^{-1} x(k+i \mid k)\right|^{2} \\
& \leq\left\|\psi_{j} \Lambda(k+i) Y_{1} \bar{M}_{1}^{-\frac{1}{2}}\right\|^{2}
\end{aligned}
$$

where $\psi_{j}$ is the $j$-th row of the $m_{-}$dimensional identity matrix. By applying the Schur complement, it is shown that LMIs (53) guarantees that $\left|u_{j}(k+i \mid k)\right| \leq \bar{u}_{j}, \quad i \geq 0, \quad j=$ $1, \ldots, m$.

If data loss happens, we assume that the number of successive data loss is $h$. Then by (41)-(43), we have

$$
\begin{aligned}
& \left|u_{j}(k+i+h+1 \mid k)\right|^{2} \\
\leq & \left\|\psi_{j} \Lambda(k+i+h+1) Y_{1} \bar{M}_{1}^{-\frac{1}{2}}\right\|^{2} \\
& \times\left\|\bar{M}_{1}^{-\frac{1}{2}} x(k+i+h+1 \mid k)\right\|^{2} \\
\leq & \left\|\psi_{j} \Lambda(k+i+h+1) Y_{1} \bar{M}_{1}^{-\frac{1}{2}}\right\|^{2},
\end{aligned}
$$

or

$$
\begin{aligned}
\left|u_{j}(k+i+h \mid k)\right|^{2} \leq & \left\|\psi_{j} \Lambda(k+i+h+1) Y_{1} \bar{M}_{1}^{-\frac{1}{2}}\right\|^{2} \\
& \times\left\|\bar{M}_{1}^{-\frac{1}{2}} x(k+i+h+1 \mid k)\right\|^{2} \\
\leq & \left\|\psi_{j} \Lambda(k+i+h) Y_{1} \bar{M}_{1}^{-\frac{1}{2}}\right\|^{2},(58)
\end{aligned}
$$

which can be transformed into (53) by Schur complement. Thus the input constraint (2) can be satisfied.

Based on all the above developments, the constrained predictive control optimization problem with data loss and quantization is formulated as follows:

$$
\min _{\left\{\varepsilon, \bar{M}_{0}, \bar{N}_{0}, \bar{M}_{1}, \bar{N}_{1}, \bar{W}_{0}, \bar{T}_{0}, \bar{W}_{1}, \bar{T}_{1}, Y_{1}, \bar{M}_{0}^{s}, \bar{N}_{0}^{s}, \bar{M}_{1}^{s}, \bar{N}_{1}^{s}\right\}} \varepsilon,
$$

s.t. (22)-(25), (38)-(39), (44)-(53) .

Theorem 1. For system (17), if the optimization problem (59) is feasible at time $k$, then it is also feasible for all $t>k$; and the closed-loop system is stochastically stable by the feasible predictive control state feedback control law in (15).

The proof is omitted here due to page length limitation.

\section{Conclusions}

In this paper, we have studied the predictive control problem for networked control systems with quantization and data loss. The proposed MPC synthesis approach not only can guarantee closed-loop mean square stability but also satisfy system constraints. Possible future extensions of this work include the analysis of the relationship between the control performance and the parameters of the network and system, especially the forgetting factor, and the adoption of a more realistic compensation strategy.

\section{References}

[1] B. Ding. Stabilization of linear systems over networks with bounded packet loss and its use in model predictive control. Automatica, 47(11):2526-2533, 2011.

[2] B. Xue, N. Li, S. Li, Q Zhu. Robust predictive control for networked control systems with quantization. IET Control Theory Appl., 4(12):2896-2906, 2010.

[3] D. E. Quevedo, J. Østergaard, D. Nešić. Packetized predictive control of stochastic systems over bit-rate limited channels with packet loss. IEEE Trans. Automat. Control, 56(12):2854-2868, 2011.

[4] D. F. Coutinho, M. Fu, C.E. de Souza. Input and output quantized feedback linear systems. IEEE Trans. Automat. Control, 55(3):761-766, 2010.

[5] H. Gao, T. Chen. A new approach to quantized feedback control systems. Automatica, 44(2):534-542, 2008.

[6] J. Xiong, J. Lam. Stabilization of linear systems over networks with bounded packet loss. Automatica, 43(1):80-87, 2007.

[7] L. Xie, L. Xie. Stability analysis of networked sampled-data linear systems with Markovian packet losses. IEEE Trans. Automat. Control, 54(6):1375-1381, 2009.

[8] M. Fu, L. Xie. The sector bound approach to quantized feedback control. IEEE Trans. Automat. Control, 50(11):16981711, 2006.

[9] M. Huang, S. Dey. Stability of Kalman filtering with markovian packet losses. Automatica, 43(4):598-607, 2007.

[10] M. Liu, D.W.C. Ho, Y. Niu. Stabilization of Markovian jump linear system over networks with random communication delay. Automatica, 45(2):416-421, 2009.

[11] N. Elia, S. Mitter. Stabilization of linear systems with limited information. IEEE Trans. Automat. Control, 46(9):13841400, 2001.

[12] S. Hu, W. Yan. Stability robustness of networked control systesms with respect to packet loss. Automatica, 43(7):12431248, 2007

[13] T. Jia, Y. Niu, Y. Zou. Sliding mode control for stochastic systems subject to packet losses. Inform. Sci., 217:117-126, 2012.

[14] X. Tang, B. Ding. Model predictive control of linear systems over networks with data quantizations and packet losses. $\mathrm{Au}$ tomatica, 49(5):1333-1339, 2013.

[15] Y. Niu, D. W. C. Ho. Design of sliding mode control subject to packet losses. IEEE Trans. Automat. Control, 55(11):2623$2628,2010$.

[16] Y. Xia, M. Fu, G. Liu. Analysis and Synthesis of Networked Control Systems. Springer-Verlag Ltd, 2011.

[17] Y. Zhao, H. Gao, T. Chen. Fuzzy constrained predictive control of nonlinear systems with packet dropouts. IET Control Theory Appl., 4(9):1665-1677, 2009.

[18] Y. Zou, Y. Niu. Predictive control of constrained linear systems with multiple missing measurements. Circuit, Syst, Sig. Proc., 32(2):615-630, 2013.

[19] Y. Zou, Y. Niu, B. Chen, T. Jia. Networked predictive control of constrained linear systems with input quantisation. Int. J. Systems Sci., 44(10):1970-1982, 2013. 